\title{
Repression of AGAMOUS-LIKE 24 is a crucial step in promoting flower development
}

\author{
Hao $\mathrm{Yu}^{1,2}$, Toshiro Ito $^{1}$, Frank Wellmer ${ }^{1}$ \& Elliot M Meyerowitz ${ }^{1}$
}

Flower development begins as floral meristems arise in succession on the flank of the inflorescence meristem. Floral meristem identity genes LEAFY (LFY) and APETALA1 (AP1) promote establishment and maintenance of floral identity in newly formed floral primordia ${ }^{1-9}$. Without their activity, the floral primordia develop with inflorescence characteristics. The underlying molecular-genetic mechanism is unknown. Here we show that these phenotypes are due in large part to the ectopic expression of AGAMOUS-LIKE 24 (AGL24), a central regulator of floral meristem identity. We present evidence that $A G L 24$ is an early target of transcriptional repression by $L F Y$ and $A P 1$. Without such repression, continued $A G L 24$ expression in floral meristems is sufficient to cause floral reversion regardless of the activation of floral organ identity genes. This indicates that $L F Y$ and AP1 promote floral development not only by positively regulating genes activated in flower development, but also by repressing $A G L 24$, a promoter of inflorescence fate.

The transition from vegetative to reproductive growth is a marked developmental change in the life of plants, whereby newly formed meristems acquire floral rather than inflorescence identity. Inflorescence meristems are indeterminate, with the production of secondary inflorescences subtended by cauline leaves, or flowers, in a spiral pattern, with internode elongation. The floral meristems that originate from inflorescence meristems are determinate, making the four types of floral organs in whorls. The fate of these meristems depends on an antagonistic interaction between the shoot identity gene TERMINAL FLOWER 1 (TFL1) and a group of floral meristem identity genes, such as $L F Y$ and AP1 (refs. 6,7,9). Previous studies have shown that TFL1 acts predominantly to oppose establishment of floral meristem identity but does not antagonize the maintenance of floral meristems $s^{6,7,10-12}$. The simple establishment of floral meristem identity is insufficient to guarantee floral identity, as indicated by the observation that a floral meristem can revert to a flower-generating inflorescence meristem, a phenomenon called floral reversion ${ }^{5,13-15}$. Floral reversion occurs for the most part in mutant genetic backgrounds, such as ap1, lfy and agamous, suggesting that wild-type plants have default processes to suppress this reversion, and that active maintenance of floral meristem identity is required after its establishment. It has been proposed that the primary effect of $L F Y$ in maintaining floral meristem identity may be transcriptional repression $^{9}$, but the potential targets are unknown.

The first indication that $A G L 24$, a member of the MADS-box family of DNA-binding transcription factors, is involved in floral meristem identity came from the phenotype of constitutive expression of AGL24. Such expression changes flowering time ${ }^{16,17}$ and causes floral reversion. 35S:AGL24 transgenic lines have a uniform, dominant and heritable alteration in flower development. The most notable aspect of this alteration is the partial reversion of floral meristems into inflorescence shoots, which was reminiscent of the effect of strong ap1 mutated alleles (Fig. 1a). In a homozygous 35S:AGL24 line, the floral meristem that would develop into a single flower in a wild-type plant often generated a central primary flower with extra secondary flowers in the axils of sepals (Fig. 1b). At later stages, the base of the ovary in a 35S:AGL24 flower elongated as an inflorescence stem would, and an ectopic inflorescence bearing flowers erupted from the developing ovary (Fig. 1d,e). Furthermore, most of the floral organs in 35S:AGL24 flowers underwent homeotic transformations toward structures normally found in inflorescence shoots (Fig. 1f-l). These phenotypes were attenuated in 35S:AGL24 hemizygotes (Fig. 1c). The observed homeotic phenotypes of 35S:AGL24 plants were not due to the cosuppression of other MADS-box family members, because both expression levels and patterns of floral organ identity genes were almost identical in 35S:AGL24 and agl24 plants (Supplementary Fig. 1 online).

The phenotype of 35S:AGL24 plants suggests that AGL24 promotes inflorescence identity. To test whether the increased inflorescence character of the lfy and ap1 mutants resulted from ectopic expression of $A G L 24$, we crossed the loss-of-function mutation agl24 into the lines with reduced $L F Y$ and $A P 1$ activity. The inflorescence phenotype of ap1-1 mutants was the appearance of ectopic flowers or inflorescences in the axils of the leaf-like first whorl floral organs, creating a supernumerary inflorescence apex (Fig. 2a,c). Floral meristems in ap1-1 showed some of the same characteristics as inflorescence meristems (Fig. 2e), to a degree that varied with their position on the main inflorescence stem (Supplementary Table 1 online). Supernumerary inflorescences were markedly suppressed in ap1-1 agl24 double mutants relative to ap1-1 single

${ }^{1}$ Division of Biology 156-29, California Institute of Technology, Pasadena, California 91125, USA. ${ }^{2}$ Department of Biological Sciences, Faculty of Science, National University of Singapore, 10 Science Drive 4, 117543 Singapore. Correspondence should be addressed to E.M.M. (meyerow@its.caltech.edu).

Published online 11 January 2004; doi:10.1038/ng1286 

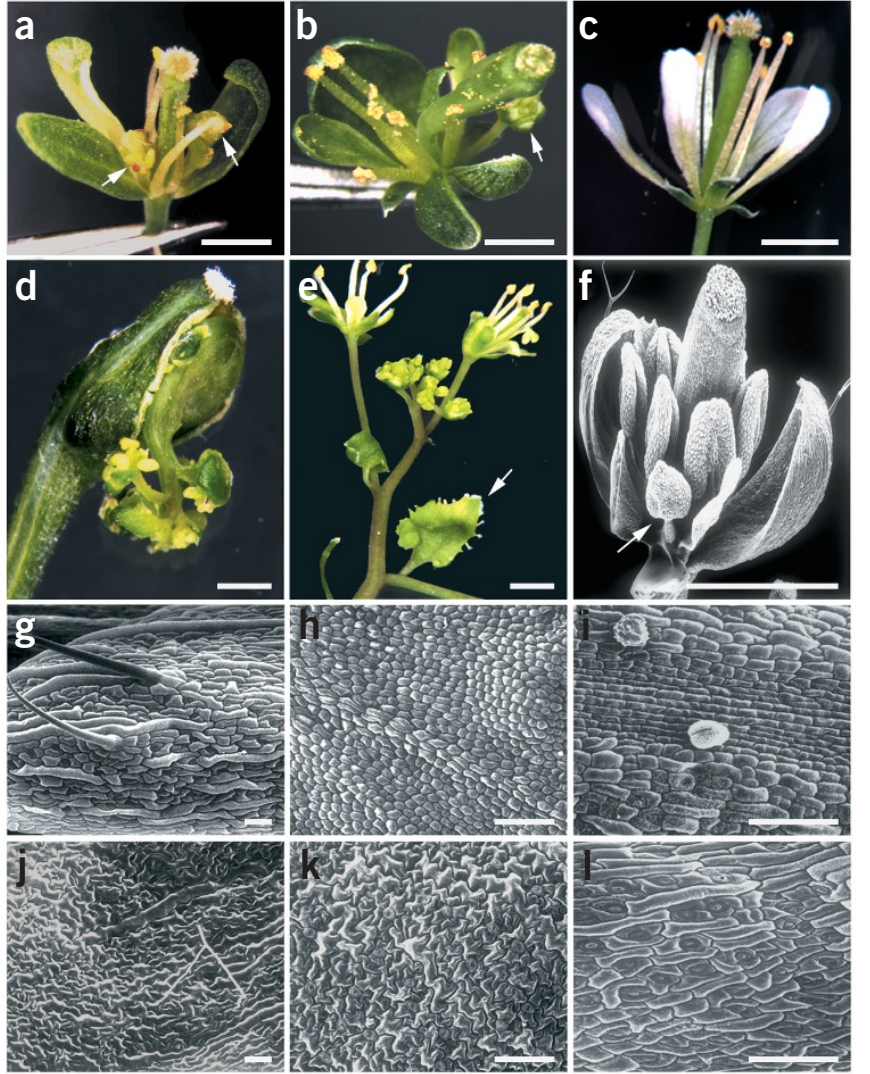

mutants (Fig. 2b,d). Most of the floral meristems in the double mutant developed as single flowers (Fig. 2f and Supplementary Table 1 online). In general, the average number of flowers produced in each pedicel or peduncle of ap1-1 agl24 mutants was comparable to that produced in wild-type plants. Some floral organ defects of ap1-1 mutants, especially the absence of petals, were also partly rescued by agl24 (Fig. 2f-h).

Loss of AGL24 function also reduced the inflorescence characteristics of $l f y-6$ mutants $^{4}$ in three ways. First, flowers in a lfy- 6 single mutant were typically subtended by bracts (Fig. 3a,c,e), as secondary inflorescence meristems in wild-type plants are. In $l f y-6$ agl24 double mutants, most flowers developed as in the wild type, without bracts (Fig. 3b,d,f). Second, floral organs were arranged in a spiral pattern in $l f y-6$ single mutants (Fig. 3e), as leaves around inflorescence meristems in wild-type plants are. But lfy-6 agl24
Figure 1 Phenotypes of constitutive expression of AGL24. (a) ap 1-1 flower had bract-like sepals and secondary flowers (arrows) without petals. (b) 35S:AGL24 flower had similar phenotypes as ap1-1 (a). Note the formation of a secondary flower (arrow). (c) 35S:AGL24/- flower had the attenuated phenotype with only bract-like sepals. (d) Ectopic inflorescence formation in the swollen ovary of a 35S:AGL24 flower. (e) Development of ectopic inflorescence shown in $\mathbf{d}$, with some carpelloid structures at the margin of cauline leaves (arrow). (f) Scanning electron micrograph (SEM) of a 35S:AGL24 flower shows stellate trichomes on the abaxial surface of the first whorl organs and the homeotic transformation of a petal into a stamen (arrow). (g-i) SEMs of the abaxial surface of a sepal (g), petal (h) and carpel (i) in a wild-type flower. (j-I) SEMs of the abaxial surface of the first whorl (j), second whorl (k) and fourth whorl (I) organs in a 35S:AGL24 flower. Scale bars: a-f, $1 \mathrm{~mm} ; \mathbf{g}-\mathbf{I}, 50 \mu \mathrm{m}$.

double mutants had floral organs largely organized in a whorled pattern (Fig. 3f). Finally, lfy-6 single mutants could have ectopic secondary flowers, but these were not seen in $l f y$ - 6 agl24 double mutants. Overall, the inflorescence architecture of a lfy- 6 agl24 double mutant more closely resembled that of a wild-type plant than that of a lfy-6 mutant (Fig. 3g). The floral organ defects in $l f y$ 6 mutants were not rescued in the lfy- 6 agl 24 double mutant, which indicates that $L F Y$ and $A P 1$ function through distinct mechanisms in the regulation of floral organ identity. Ify-6 ap1-1 double mutants showed enhanced inflorescence phenotypes as compared with single mutants ${ }^{4}$, and these were rescued in an agl24 background, as in the single mutants (data not shown). These genetic data indicate that AGL24 contributes much of the inflorescence character of $l f y$ and apl mutants, supporting the possibility that $L F Y$ and $A P 1$ act to repress AGL24 in wild-type plants.

To test directly the potential repression of AGL24 by the floral meristem identity genes, we carried out in situ hybridization with the AGL24 probe to lfy-6 and ap1-1 inflorescences. In wild-type plants during their vegetative phase, AGL24 is expressed in the entire shoot apical meristem and in emerging leaf primordia ${ }^{16}$. During floral transition, AGL24 RNA was detectable in the transitional shoot apex and in young cauline leaves (Fig. 4d). AGL24 expression in early floral meristems differed from that in inflorescence meristems. In floral meristems from stages 1 to $4, A G L 24$ was expressed only in the tunica, the superficial layers of the meristems (Fig. 4a). The absence of AGL24 RNA in the corpus of floral primordia coincided with the developmental stages at which $L F Y$ and $A P 1$ are expressed in the floral primordia ${ }^{4,18}$. Thus, in the corpus but not in the tunica, the activity of $L F Y$ and $A P 1$ may repress $A G L 24$. At later stages of floral development, $A G L 24$ RNA was weakly expressed in the distal parts of stamens and carpels (Fig. $\mathbf{4 b}$ ). Its expression was restricted to pollen and the adaxial surface of the gynoecium after floral stage 9 (Fig. 4c).

\begin{abstract}
Figure 2 Rescue of ap1-1 by ag/24. Light micrographs of ap 1-1 (a,c,e,g) and ap 1-1 agl24 $(\mathbf{b}, \mathbf{d}, \mathbf{f}, \mathbf{h})$ mutants are shown. (a,b) Top view of a developing inflorescence. (c,d) Side view of a developing inflorescence. $(\mathbf{e}, \mathbf{f})$ Side view of a floral structure arising from an individual floral meristem at a basal position in the main inflorescence. (g,h) Side view of a flower arising at an apical position in the main inflorescence.
\end{abstract}
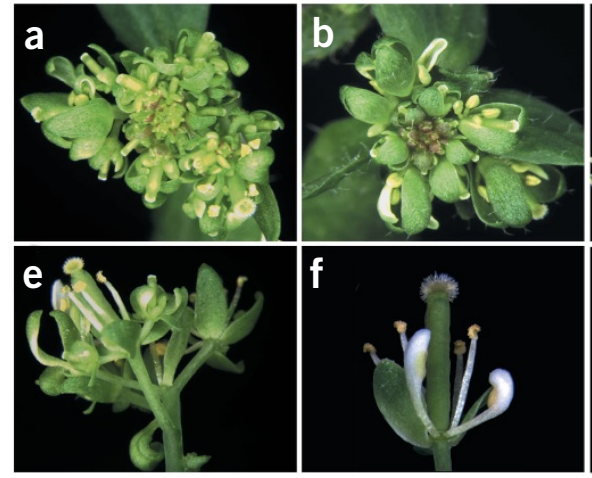

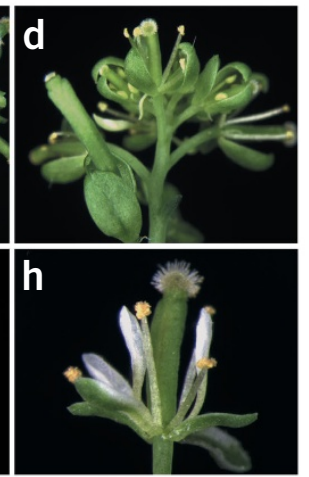



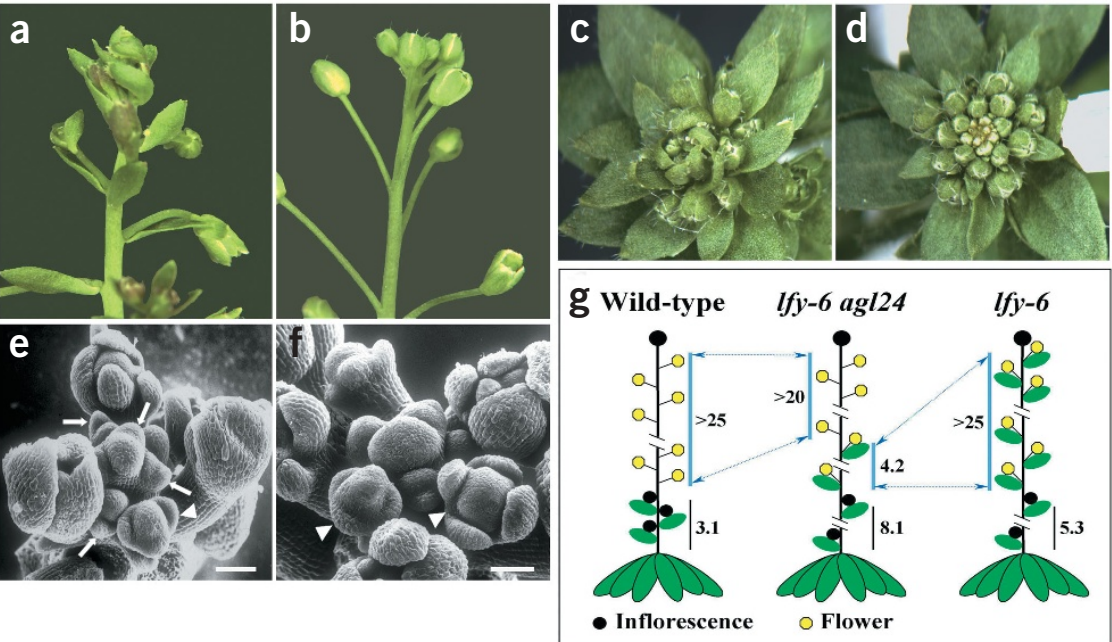

Figure 3 Rescue of Ify- 6 by ag/24. Phenotypes of Ify- 6 (a,c,e) and Ify- 6 ag/24 (b,d,f) mutants are shown. (a,b) Side view of a developing inflorescence. (c,d) Top view of a developing inflorescence. The cauline leaf-like bracts subtending flowers in Ify- 6 mutants $(\mathbf{a}, \mathbf{c})$ were lost in Ify- 6 ag/24 mutants (b,d). (e,f) SEM of an inflorescence apex. Bract structures (arrows) surrounding floral meristems in Ify- 6 mutants (e) disappeared in Ify- 6 ag/24 mutants (f), and the spiral mode of floral organ formation (arrowhead) in Ify- 6 mutants became the whorled mode (arrowhead). (g) Schematic representation of the rescue of Ify- 6 by ag/24. The mean numbers of different structures calculated from 20 samples are given. The Ify- 6 ag/24 double mutant produced around eight secondary inflorescences, four flowers subtended by bracts, as in Ify- 6 mutants, and the rest of the apical flowers without bracts, as in a wild-type plant. Scale bars: e,f, $100 \mu \mathrm{m}$.
In ap1-1 mutants, AGL24 was ectopically expressed in the corpus of the young floral primordia (Fig. 4e), and its strong expression was persistent in all of the floral organs at later stages of flower development (Fig. 4f,g). We observed similar ectopic expression of AGL24 in the supernumerary meristems of ap 1-1 cal-1 mutants (Fig. $4 \mathbf{h}$ ), indicating that ectopic expression of AGL24 was responsible for the enhanced inflorescence traits in ap 1-1 cal-1 mutants. In lfy-6 mutants, ectopic expression of AGL24 was also apparent throughout the young floral meristems (Fig. 4i), which coincided with the reduced expression of $A P 1$ RNA in the same region (Fig. $4 \mathbf{m}, \mathbf{n}$ ). The later expression of AP1 in $l f y-6$ mutants is comparable to that in the wild type (Fig. 4o,p). The ectopic expression of AGL24 in emerging floral primordia and the corresponding cauline leaves in an lfy-6 mutant (Fig. 4l) seemed stronger than its expression in the transitional inflorescence meristem of a wild-type plant (Fig. 4d). This indicates that floral primordia in $l f y-6$ mutants initiated with some degree of inflorescence character. AGL24 RNA in $l f y-6$ mutants was also distributed in floral organ primordia (Fig. 4j), predominantly in the irregularly fused carpels at later stages (Fig. 4k). It is therefore possible to conclude that the increase of inflorescence traits in flowers of $a p 1-1$ and $l f y-6$ mutants, which mimics the phenotypes of gain of function of AGL24, resulted from ectopic expression of AGL24.

To further confirm that $L F Y$ and $A P 1$ act as transcriptional repressors of AGL24, we used steroid-inducible versions of AP1 and LFY in transgenic plants ${ }^{19}$. Before using the AP1-GR construct, we tested its biological activity. The development of 35S:AP1-GR transformants was indistinguishable from that of wild-type plants in the absence of applied steroid. After treatment with dexamethasone, both the primary and secondary inflorescences of 35S:AP1-GR plants terminated as floral meristems and formed terminal flowers, which is characteristic of AP1
Figure 4 In situ localization of AGL24 (a-I) and $A P 1$ ( $\mathbf{m}-\mathbf{p})$ RNA. (a-d) AGL24 expression in wild-type plants. AGL24 was expressed in the inflorescence meristem and the tunica region of young floral meristems (a and inset), in the distal region of stamen and carpel primordia (b) and later in the pollen and the adaxial surface of the gynoecium (c). During the floral transition, AGL24 RNA was distributed in the shoot apex and the corresponding cauline leaves (d). (e-g) AGL24 expression in ap1-1. AGL24 was ectopically expressed in the whole zone of floral meristems (e) as well as the stamen and carpel primordia (f), and later in the petals, anthers and gynoecium (g). (h) Ectopic AGL24 expression in the supernumerary meristems in ap 1-1 cal-1. (i-I) AGL24 expression in Ify-6. AGL24 was ectopically expressed in the whole zone of floral meristems (i) and several organ primordia ( $\mathbf{j}$ ), and later mainly confined to the central region of the flower (k). AGL24 expression was stronger in the young floral primordium and the coupled cauline leaf-like bract (I) than in the transitional inflorescence apex (d). $(\mathbf{m}-\mathbf{p}) A P 1$ expression in $1 f y-6(\mathbf{m}, \mathbf{0})$ and wild-type plants $(\mathbf{n}, \mathbf{p})$. In Ify- 6 , the initial expression of $A P I$ was much lower ( $\mathbf{m})$ than in the wild-type plant, and its later expression (o) was comparable with that in the wild-type plant (p). Scale bars, $100 \mu \mathrm{m}$.
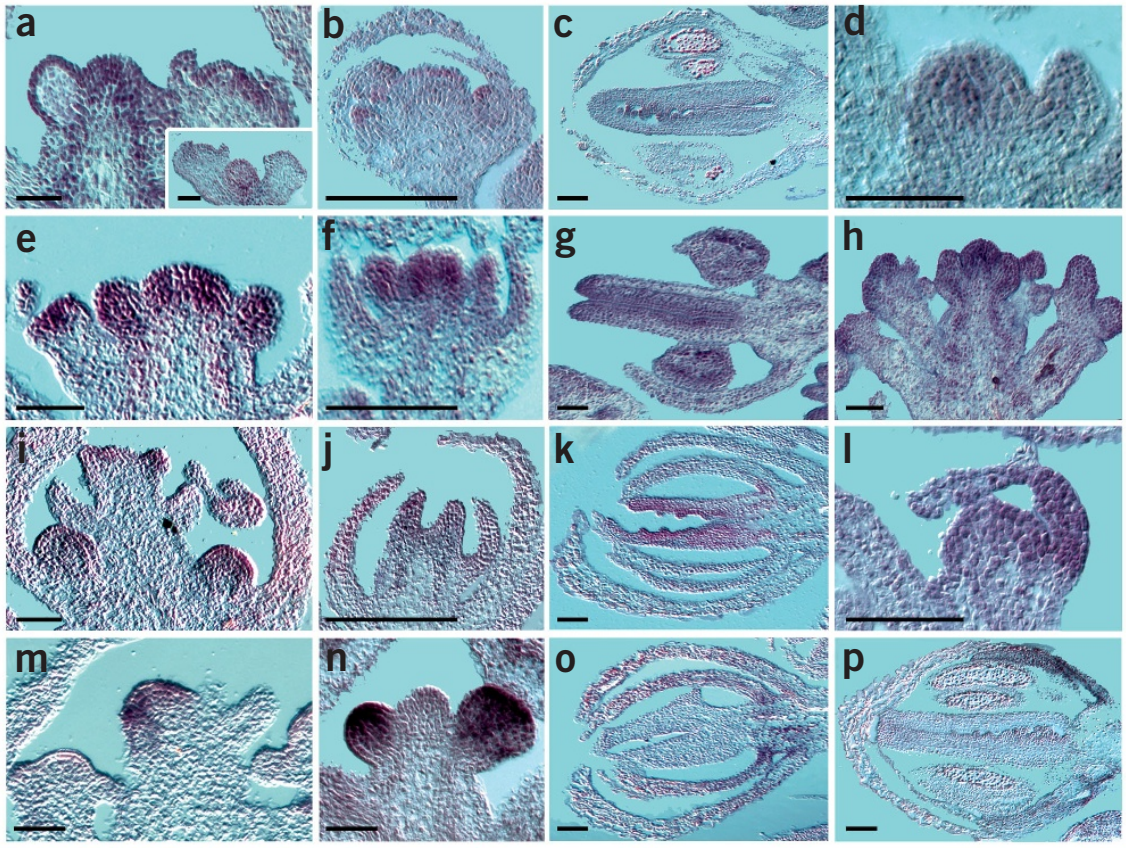
Figure 5 Transcriptional repression of $A G L 24$ by $A P 1$ and $L F Y$. (a-d) A biologically active $A P 1-G R$ fusion. Individual seedlings of 7-day-old ap 1-1 35S:AP1-GR plants were mock-treated $(0.03 \%$ ethanol and $0.015 \%$ Silwet L-77) or treated with $10 \mu \mathrm{M}$ dexamethasone and $0.015 \%$ Silwet L-77 twice with a one-week interval between treatments. Sixteen days after the first treatment, mock-treated plants had the same inflorescences as ap 1-1 mutants (a), whereas dexamethasonetreated plants had a rescued phenotype with terminal flowers (b), mimicking 35S:AP1 plants. The rescue of floral organ defects, such as the generation of sepals and petals, was obvious in late-stage inflorescences of dexamethasonetreated ap1-1 35S:AP1-GR plants (c), in which most flowers were fully rescued (d). (e) Timecourse expression of AGL24 in inflorescences of ap1-1 35S:AP1-GR and Ify-6 35S:LFY-GR plants mock-treated $(\mathrm{M})$ or treated with $10 \mu \mathrm{M}$ dexamethasone (D). (f) Expression of AGL24 and AP1 in inflorescences of Ify- $635 S$ :LFY-GR plants, and expression of AGL24 in inflorescences of ap1-1 35S:AP1-GR plants. The inflorescences were mock-treated (M) or treated with $10 \mu \mathrm{M}$ dexamethasone (D), with $10 \mu \mathrm{M}$ cycloheximide (C) or with $10 \mu \mathrm{M}$ cycloheximide plus dexamethasone (CD). Expression analyses were done after $4 \mathrm{~h}$ of treatment. In (e) and (f), the numbers below each lane indicate the relative expression levels of each studied gene, calculated by normalizing each signal first against the signal for TUB2 and then against the first-lane value. gain-of-function plants $^{20}$. Activation of $35 S: A P 1-G R$ in an ap1-1 genetic background resulted in partial or full rescue of ap1-1 mutant phenotypes (Fig. 5a-d), suggesting that the AP1-GR fusion was biologically functional in a hormone-dependent manner.

Treatment with dexamethasone of inflorescence apices of ap1-1 35S:AP1-GR plants for $4 \mathrm{~h}$ or longer resulted in a reduction of AGL24 RNA level relative to mock-treated controls (Fig. 5e). A combined treatment of dexamethasone and cycloheximide, an inhibitor of translation, at the 4 -h time point resulted in a similar reduction of AGL24 RNA as observed with dexamethasone treatment alone (Fig. 5f), indicating that AGL24 may be an immediate target of transcriptional repression by $A P 1$. We also investigated AGL24 expression in the established 35S:LFY-GR system ${ }^{19}$. AGL24 RNA level was decreased after treatment of lfy-6 35S:LFY-GR plants

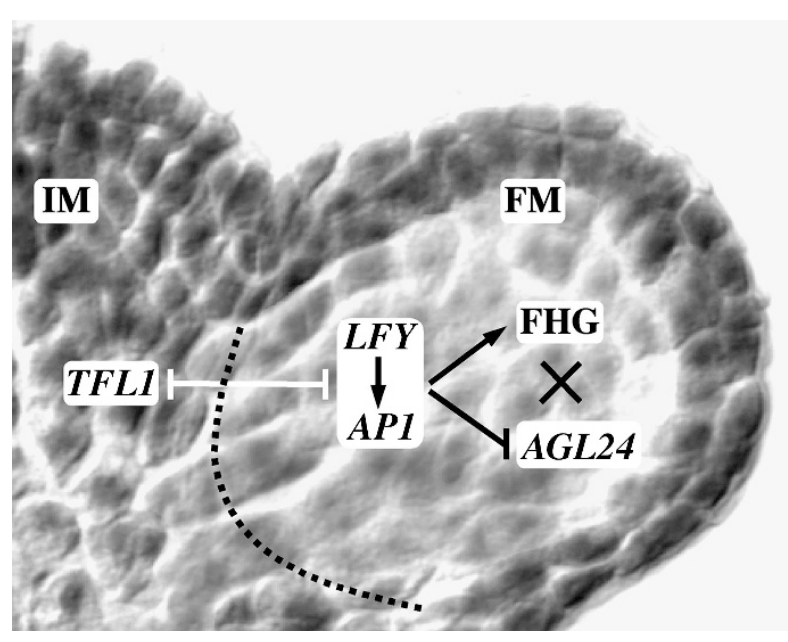

Figure 6 Model for floral meristem development. $L F Y$ and $A P 1$ antagonize TFL 1 activity to establish floral meristem (FM) identity on the flanks of the inflorescence meristem (IM) and repress AGL24 to prevent its interference with the normal functions of floral homeotic genes (FHG). AGL24 expression is shown in the background, where the putative border between the inflorescence meristem and the floral meristem is indicated as a dotted line. with dexamethasone for $4 \mathrm{~h}$ or more (Fig. 5e), suggesting that $L F Y$ also acts as a repressor of AGL24. But we did not observe similar repression after a combined treatment with dexamethasone and cycloheximide (Fig. 5f), indicating that this repression may be modulated by other mediators that require translation after $L F Y$ activation, which may include AP1.

Taken together, the results presented here suggest that AGL24 is among the central regulators of floral meristem development, acting to promote inflorescence identity, and is repressed by the floral meristem identity genes $L F Y$ and $A P 1$. This negative interaction identifies two antagonistic genetic pathways in flower development (Fig. 6), in which $L F Y$ and AP1 not only activate the ABC floral organ identity genes to promote flower development ${ }^{21-23}$, but also repress AGL24 to secure the maintenance of floral rather than inflorescence meristem identity.

\section{METHODS}

Plant materials. All mutations used in this study are in the Landsberg erecta (Ler) background except when stated otherwise. We grew them at $22^{\circ} \mathrm{C}$ in continuous light. We obtained the Ler near-isogenic agl24 line by three backcrosses of the agl24 Columbia line into Ler ${ }^{16}$. We identified the lfy-6 agl24 and ap1-1 agl24 double mutants by screening for mutant phenotypes and confirmed their identification by PCR genotyping of all three genes. We genotyped agl24, lfy-6, and $a p 1-1$ as reported previously $8,17,24$. We screened 35S:AP1-GR plants by Basta selection and further tested them for phenotypic effects after dexamethasone treatment. We selected one transgenic line, which contains only one transgene insertion and shows the phenotype closely resembling 35S:AP1 transformants ${ }^{20}$ after hormone induction, to cross with ap 1-1 to generate ap11 35S:AP1-GR plants. The selection of 35S:AGL24 plants has been described elsewhere ${ }^{16}$.

Plasmid constructs. The construction of 35S:AGL24 is described previously ${ }^{16}$. For 35S:AP1-GR, we constructed a derivative pGreen-35S vector by cloning a $35 \mathrm{~S}$ promoter cassette from p35S-2 into the EcoRV and StuI sites of pGREEN0229 (ref. 25). We amplified the hormone-binding domain of the rat glucocorticoid receptor (GR) by PCR from $\mathrm{pBI}-\Delta \mathrm{GR}^{26}$. We removed an internal EcoRI site in this GR fragment by silent mutagenesis and cloned the resulting fragment into pBSII-SK (Stratagene). We released the GR fragment from pBSII-SK at the SacI and EcoRI sites and cloned it into pGREEN0229-35S to generate pGREEN0229-35S:GR. We amplified the AP1 coding region ${ }^{18}$ by PCR and cloned it into the vector pZERO blunt II TOPO (Invitrogen). We then cut 
out the AP1 fragment with $\mathrm{XbaI}$ and BamHI and inserted it into pGREEN022935S:GR to yield pGREEN0229-35S:AP1-GR. We sequenced the construct to detect potential PCR-introduced mutations.

Expression analyses. Dexamethasone treatment and sample collection were previously described ${ }^{19}$. We extracted total RNA by RNeasy Plant Mini Kit (Qiagen) and reverse-transcribed it using the ThermoScript RT-PCR system (Invitrogen). We carried out RT-PCR and then detected expression by Southern-blot analysis as described previously ${ }^{16}$. We synthesized antisense RNA probes for in situ hybridization from plasmids pHY301(AGL24 $)^{16}$, pDW124(LFY $)^{4}$, pAM128 $(A P 1)^{18}, \mathrm{pD} 793(A P 3)^{27}$ and pCIT565 $(A G)^{28}$. We carried out nonradioactive in situ hybridization according to a published protocol ${ }^{29}$.

Scanning electron microscopy. We prepared samples as described ${ }^{30}$.

Note: Supplementary Information is available on the Nature Genetics website.

\section{ACKNOWLEDGMENTS}

We thank P.P. Kumar and members of the laboratory of E.M.M. for comments. This work was supported by a grant from the US National Institutes of Health (to E.M.M.). H.Y. was supported by an overseas postdoctoral fellowship from National University of Singapore and a grant from the US National Institutes of Health (to E.M.M.)

\section{COMPETING INTERESTS STATEMENT}

The authors declare that they have no competing financial interests.

Received 3 November; accepted 2 December 2003

Published online at http://www.nature.com/naturegenetics/

1. Irish, V.F. \& Sussex, I.M. Function of the apetala-1 gene during Arabidopsis flora development. Plant Cel/ 2, 741-753 (1990).

2. Schultz, E.A. \& Haughn, G.W. LEAFY, a homeotic gene that regulates inflorescence development in Arabidopsis. Plant Cel/ 3, 771-781 (1991).

3. Huala, E. \& Sussex, I.M. LEAFY Interacts with Floral Homeotic Genes to Regulate Arabidopsis Floral Development. Plant Cel/ 4, 901-913 (1992).

4. Weigel, D., Alvarez, J., Smyth, D.R., Yanofsky, M.F. \& Meyerowitz, E. M. LEAFY controls floral meristem identity in Arabidopsis. Cel/ 69, 843-859 (1992).

5. Bowman, J.L., Alvarez, J., Weigel, D., Meyerowitz, E.M. \& Smyth, D.R. Control of flower development in Arabidopsis thaliana by APETALA1 and interacting genes. Development 119, 721-743 (1993).

6. Shannon, S. \& Meeks-Wagner, D.R. Genetic interactions that regulate inflorescence development in Arabidopsis. Plant Cel/ 5, 639-655 (1993).

7. Ratcliffe, O.J., Bradley, D.J. \& Coen, E.S. Separation of shoot and floral identity in Arabidopsis. Development 126, 1109-1120 (1999).

8. Ferrandiz, C., Gu, Q., Martienssen, R. \& Yanofsky, M.F. Redundant regulation of meristem identity and plant architecture by FRUITFULL, APETALA1 and CAULI-
FLOWER. Development 127, 725-734 (2000)

9. Parcy, F., Bomblies, K. \& Weigel, D. Interaction of LEAFY, AGAMOUS and TERMINAL FLOWER1 in maintaining floral meristem identity in Arabidopsis. Development 129 2519-2527 (2002).

10. Shannon, S. \& Meeks-Wagner, D.R. A mutation in the Arabidopsis TFL1 gene affects inflorescence meristem development. Plant Cel/ 3, 877-892 (1991).

11. Bradley, D., Ratcliffe, O., Vincent, C., Carpenter, R. \& Coen, E. Inflorescence commitment and architecture in Arabidopsis. Science 275, 80-83 (1997).

12. Ratcliffe, O.J. et al. A common mechanism controls the life cycle and architecture of plants. Development 125, 1609-1615 (1998).

13. Clark, S.E., Running, M.P. \& Meyerowitz, E.M. CLAVATA1, a regulator of meristem and flower development in Arabidopsis. Development 119, 397-418 (1993).

14. Okamuro, J.K., den Boer, B.G. \& Jofuku, K.D. Regulation of Arabidopsis flower development. Plant Cel/ 5, 1183-1193 (1993).

15. Mizukami, Y. \& Ma, H. Determination of Arabidopsis floral meristem identity by AGA MOUS. Plant Cel/ 9, 393-408 (1997).

16. Yu, H., Xu, Y., Tan, E.L. \& Kumar, P.P. AGAMOUS-LIKE 24, a dosage-dependent mediator of the flowering signals. Proc. Natl. Acad. Sci. USA 99, 16336-16341 (2002)

17. Michaels, S.D. et al. AGL24 acts as a promoter of flowering in Arabidopsis and is positively regulated by vernalization. Plant J. 33, 867-874 (2003).

18. Mandel, M.A., Gustafson-Brown, C., Savidge, B. \& Yanofsky, M.F. Molecular characterization of the Arabidopsis floral homeotic gene APETALA1. Nature 360, 273-277 (1992).

19. Wagner, D., Sablowski, R.W. \& Meyerowitz, E.M. Transcriptional activation of APETALA1 by LEAFY. Science 285, 582-584 (1999).

20. Mandel, M.A. \& Yanofsky, M.F. A gene triggering flower formation in Arabidopsis Nature 377, 522-524 (1995).

21. Parcy, F., Nilsson, O., Busch, M.A., Lee, I. \& Weigel, D. A genetic framework for floral patterning. Nature 395, 561-566 (1998).

22. Ng, M. \& Yanofsky, M.F. Activation of the Arabidopsis B class homeotic genes by APETALA1. Plant Cell 13, 739-753 (2001).

23. Weigel, D. \& Meyerowitz, E.M. The ABCs of floral homeotic genes. Cel/ 78, 203-209 (1994).

24. Blazquez, M.A., Soowal, L.N., Lee, I. \& Weigel, D. LEAFY expression and flower initiation in Arabidopsis. Development 124, 3835-3844 (1997).

25. Hellens, R., Edwards, E., Leyland, N., Bean, S. \& Mullineaux, P. pGreen: a versatile and flexible binary Ti vector for Agrobacterium-mediated plant transformation. Plant Mol. Biol. 42, 819-832 (2000).

26. Lloyd, A.M., Schena, M., Walbot, V. \& Davis, R.W. Epidermal cell fate determination in Arabidopsis: patterns defined by a steroid-inducible regulator. Science 266, 436-439 (1994).

27. Jack, T., Brockman, L.L. \& Meyerowitz, E.M. The homeotic gene APETALA3 of Arabidopsis thaliana encodes a MADS box and is expressed in petals and stamens. Cell 68, 683-697 (1992).

28. Drews, G.N., Bowman, J.L. \& Meyerowitz, E.M. Negative regulation of the Arabidopsis homeotic gene AGAMOUS by the APETALA2 product. Cell 65, 991-1002 (1991).

29. Long, J.A. \& Barton, M.K. The development of apical embryonic pattern in Arabidopsis. Development 125, 3027-3035 (1998).

30. Bowman, J.L., Smyth, D.R. \& Meyerowitz, E.M. Genetic interactions among floral homeotic genes of Arabidopsis. Development 112, 1-20 (1991). 